\title{
BMJ Open Prevalence of retinopathy in prediabetes: protocol for a systematic review and meta-analysis
}

\author{
Varo Kirthi (1) ,1,2 Paul Nderitu, , ${ }^{1,2}$ Uazman Alam,, ${ }^{3,4,5}$ Jennifer Evans (1) ,6 \\ Sarah Nevitt (1) , ${ }^{7}$ Rayaz A Malik (D) , ${ }^{8}$ Timothy L Jackson (D) ${ }^{1,2}$
}

To cite: Kirthi V, Nderitu P, Alam U, et al. Prevalence of retinopathy in prediabetes: protocol for a systematic review and meta-analysis. BMJ Open 2021;11:e040997. doi:10.1136/ bmjopen-2020-040997

- Prepublication history supplemental material for this paper is available online. To view these files, please visit the journal online (http://dx.doi. org/10.1136/bmjopen-2020040997).

Received 28 May 2020 Revised 08 August 2020 Accepted 29 October 2020

Check for updates

(C) Author(s) (or their employer(s)) 2021. Re-use permitted under CC BY-NC. No commercial re-use. See rights and permissions. Published by BMJ.

For numbered affiliations see end of article.

Correspondence to

Dr Varo Kirthi; v.kirthi@nhs.net

\section{ABSTRACT}

Introduction There is growing evidence of a higher than expected prevalence of retinopathy in prediabetes. This paper presents the protocol of a systematic review and meta-analysis of retinopathy in prediabetes. The aim of the review is to estimate the prevalence of retinopathy in prediabetes and to summarise the current data. Methods and analysis This protocol is developed in accordance with the Preferred Reporting Items for Systematic Reviews and Meta-Analyses Protocols (PRISMA-P) guidelines. A comprehensive electronic bibliographic search will be conducted in MEDLINE, EMBASE, Web of Science, Cumulative Index to Nursing and Allied Health Literature (CINAHL), Google Scholar and the Cochrane Library. Eligible studies will report prevalence data for retinopathy on fundus photography in adults with prediabetes. No time restrictions will be placed on the date of publication. Screening for eligible studies and data extraction will be conducted by two reviewers independently, using predefined inclusion criteria and prepiloted data extraction forms. Disagreements between the reviewers will be resolved by discussion, and if required, a third (senior) reviewer will arbitrate. The primary outcome is the prevalence of any standard features of diabetic retinopathy (DR) on fundus photography, as per International Clinical Diabetic Retinopathy Severity Scale (ICDRSS) classification. Secondary outcomes are the prevalence of (1) any retinal microvascular abnormalities on fundus photography that are not standard features of DR as per ICDRSS classification and (2) any macular microvascular abnormalities on fundus photography, including but not limited to the presence of macular exudates, microaneurysms and haemorrhages. Risk of bias for included studies will be assessed using a validated risk of bias tool for prevalence studies. Pooled estimates for the prespecified outcomes of interest will be calculated using random effects meta-analytic techniques. Heterogeneity will be assessed using the $\mathrm{I}^{2}$ statistic.

Ethics and dissemination Ethical approval is not required as this is a protocol for a systematic review and no primary data are to be collected. Findings will be disseminated through peer-reviewed publications and presentations at national and international meetings including Diabetes UK, European Association for the Study of Diabetes, American Diabetes Association and International Diabetes Federation conferences. PROSPERO registration number CRD42020184820.

\section{Strengths and limitations of this study}

This systematic review protocol follows the Preferred Reporting Items for Systematic Review and MetaAnalyses Protocols (PRISMA-P) guidelines.

- This systematic review addresses an important gap in the current evidence by estimating the prevalence of retinopathy in prediabetes.

- There is potential for significant clinical and statistical heterogeneity in the reporting of prevalence data between different populations.

\section{BACKGROUND}

Prediabetes is defined by blood glucose levels above the normal range but below the threshold for type 2 diabetes mellitus. ${ }^{12}$ The burden of prediabetes is enormous: it is currently estimated toaffect 373 million people across the globe and this number is projected to increase to 587 million $(8.3 \%$ of the global adult population) by $2045 .^{3}$

Cohort analyses of people with prediabetes reveals an increased incidence of microvascular and macrovascular disease, including an elevated all-cause mortality, compared to people with normal glucose metabolism. ${ }^{45}$ This suggests that end-organ complications of hyperglycaemia may be occurring prior to the onset of overt diabetes. ${ }^{6}$ Furthermore, people with prediabetes and microvascular disease are more likely to develop overt diabetes. ${ }^{78}$ In a population-based analysis of 49072 people with diabetes, the presence of diabetic retinopathy (DR) was associated with an increased risk (HR 1.39, 95\% CI 1.09 to 1.76 ) of cardiovascular death, non-fatal myocardial infarction or stroke, after adjustment of traditional risk factors including $\mathrm{HbAlc}$, lipid profile and blood pressure. ${ }^{9}$ Despite ongoing debate on how best to identify people with prediabetes at high risk of end-organ complications, long-term data show a reduction in both morbidity and mortality following early lifestyle interventions. ${ }^{10}$ 
A systematic review of 35 population-based studies of people with diabetes reported the prevalence of DR, proliferative DR, diabetic macular oedema and visionthreatening DR as $34.6 \%, 7.0 \%, 6.8 \%$ and $10.2 \%$, respectively. ${ }^{11}$ The early onset of retinopathy in prediabetes is of particular concern as DR remains one of the principal causes of vision loss in adults of working age in developed countries, with considerable health and socioeconomic consequences. ${ }^{12}$ Given projections that up to $70 \%$ of people with prediabetes may eventually develop diabetes during their lifespan, early identification of retinopathy is a significant health priority. ${ }^{6}$ It is estimated that up to $95 \%$ of vision loss in diabetes is preventable or treatable, if detected early. ${ }^{13}$

Previous studies have suggested that isolated retinopathy changes occur in 5\%-10\% of the general population and in $2.6 \%-8.6 \%$ of those without diabetes or hypertension. ${ }^{14} 15$ Although several studies have reported retinopathy changes in prediabetes, there has been no systematic review or meta-analysis of the literature to estimate an overall prevalence. Establishing the prevalence of retinopathy may not only focus attention on early interventions but also help refine diagnostic criteria and risk stratification for prediabetes. The aim of this systematic review is to estimate the prevalence of retinopathy detected on fundus photography in adults with prediabetes.

\section{METHODS AND ANALYSIS \\ Study design}

Comprehensive literature searches of electronic bibliographic databases will be conducted in MEDLINE (access via OVID), EMBASE (access via OVID), Web of Science, CINAHL (Cumulative Index to Nursing and Allied Health Literature), Google Scholar and the Cochrane Library. No time restrictions will be placed on the date of publication. All search strategies will be independently reviewed by an expert information specialist using the Peer Review of Electronic Search Strategies checklist, and a draft MEDLINE search strategy is included in online supplemental appendix $1 .{ }^{16}$ Additional articles will be identified by searching the references of included studies and other review articles identified during the course of the searches. Results from the database searches will be merged using an electronic reference manager to facilitate removal of duplicates. Trial registries such as ClinicalTrials.gov will be consulted to identify studies that may not have been indexed in the databases. Relevant publications will be retrieved manually if electronic access is not available.

\section{Participants, eligibility and setting}

Inclusion criteria will be adults over 18 years of age who have prediabetes defined by American Diabetes Association (ADA) criteria. ${ }^{1}$ This includes impaired fasting glucose (IFG) and impaired glucose tolerance (IGT) as subgroups of prediabetes. Population-based cohort or cross-sectional studies from any country in any setting will be considered, provided they have been reported in English. Studies must report prevalence data for retinopathy detected on fundus photography, using any accepted method (eg, 1-field, 2-field, 3-field or 7-field dilated stereoscopic colour fundus photography) at least once in the study population. A lack of detail on the method used or quality of images taken will be documented but will not be considered an exclusion criterion. Studies that report other methods of imaging, such as fluorescein angiography or optical coherence tomography, will be included only if fundus photography data are also provided. Use of alternative diagnostic criteria for prediabetes, such as World Health Organization (WHO) criteria, will be recorded and prevalence figures reported separately but will not be considered a reason for exclusion. ${ }^{2}$ A lack of reporting of the definition of prediabetes and/or retinopathy will be documented but will not be considered a reason for exclusion.

\section{Outcomes}

The primary outcome is the prevalence of any standard features of DR on fundus photography, as per International Clinical Diabetic Retinopathy Severity Scale (ICDRSS) classification. ${ }^{17}$ This will be defined by the presence of any of the following features:

- Microaneurysms.

- Intraretinal haemorrhages.

- Hard exudates.

- Cotton-wool spots.

- Venous beading.

- Intraretinal microvascular abnormalities (IRMA).

- New vessels at the optic disc (NVD) or elsewhere (NVE).

- Vitreous or preretinal haemorrhage.

Secondary outcomes are the prevalence of: (1) any retinal microvascular abnormalities on fundus photography that are not standard features of DR as per ICDRSS classification and (2) any macular microvascular abnormalities on fundus photography, including but not limited to the presence of macular exudates, microaneurysms or haemorrhages.

If available, data on glycaemic parameters such as fasting glucose, 2-hour oral glucose tolerance test and HbA1c will be extracted. Similarly, if reported, prevalence data on cardiovascular parameters such as systolic and diastolic blood pressure, lipid profile and metabolic syndrome will also be extracted. Metabolic syndrome will be defined as per consensus criteria based on WHO, National Cholesterol Education Program Adult Treatment Panel III and ADA classifications. ${ }^{218-21}$

\section{Study selection}

Two reviewers will independently screen titles and abstracts from the searches and exclude any that clearly do not satisfy the inclusion criteria. Any disagreements will be resolved by discussion, and if required, a third (senior) reviewer will arbitrate. Articles of interest will be selected for a full-text assessment. If there is any doubt 
regarding the eligibility of a study, the article will be selected for full-text assessment.

Two reviewers will independently assess the full-text articles against the eligibility criteria. Disagreements between these reviewers will be resolved by discussion, and if required, a third (senior) reviewer will arbitrate.

A Preferred Reporting Items for Systematic Reviews and Meta-Analyses (PRISMA) flowchart of the selection process will be included in the systematic review. ${ }^{22}$

\section{Data collection process}

Two reviewers will independently extract data in duplicate using pre-piloted forms. Data recorded will include: (1) date and country of study; (2) study design; (3) age, gender and ethnicity of participants; (4) definition of retinopathy and method(s) used to obtain images; (5) definition of prediabetes and method(s) used to make diagnosis; (6) study groups and sizes; (7) overall sample size and (8) prevalence number and estimate. If present, secondary outcome data will also be recorded, including (1) definition and prevalence of non-standard retinopathy features and (2) definition and prevalence of maculopathy features. Where reported, prevalence estimates for comorbid ocular pathology (eg, cataract) and cardiovascular risk factors (eg, hypertension, metabolic syndrome) will also be recorded.

\section{Risk of bias assessment}

A modified critical appraisal tool for specifically assessing risk of bias in prevalence studies will be used on selected articles and is included in online supplemental appendix $2{ }^{23}$ The tool includes 9 questions, each scoring 0 or 1 , to determine confounding, selection bias and bias related to measurement and data analysis. Overall risk of bias will be determined by the total score for each article: $0-3$ considered low risk, 4-6 considered moderate risk and $\geq 7$ considered high risk. Quality assessment will be undertaken by two reviewers independently. Disagreements will be resolved by discussion, and if required, a third (senior) reviewer will arbitrate. Judgements on the overall risk of bias will be categorised as either low, moderate or high risk, based on the risk of bias of the 10 individual items listed within the tool.

\section{Data analysis}

Data will be analysed using purpose-built software for systematic reviews and meta-analyses (Review Manager V.5). Heterogeneity between included studies will be assessed based on study design, populations and methods used to measure outcomes. Statistical heterogeneity will be assessed using the $\mathrm{I}^{2}$ statistic and by visual inspection of forest plots.

Characteristics of included studies will be presented in summary tables and narrative text. In expectation of prevalence varying between studies and populations, pooled prevalence estimates for the prespecified outcomes of interest will be calculated applying random effects metaanalytic methods and reported in forest plots.
Where clinical and/or statistical heterogeneity is deemed too large by the reviewers (eg, $\mathrm{I}^{2} \geq 90 \%$ ), a systematic review without meta-analysis will be reported. Narrative synthesis will be conducted where quantitative data required for meta-analysis is lacking or absent.

Depending on availability of data, subgroup analyses using the following covariates will also be considered:

- WHO region or country.

- Age group (eg, 18-30, 31-50, >50 years).

- Ethnicity (especially at-risk groups, eg, South Asian, African, Afro-Caribbean, Hispanic).

- Time since diagnosis of prediabetes (eg, $<1$ year, $1-5$ years, $6-10$ years, $>10$ years).

- Subtype of prediabetes (eg, IFG compared with IGT).

- Grade of retinopathy as per ICDRSS classification.

- Comorbid ocular pathology (eg, cataract).

- Comorbid cardiovascular risk factors (eg, hypertension, metabolic syndrome).

- Method or criteria used to diagnose prediabetes (eg, WHO).

- Method used to diagnose retinopathy (eg, 7-field stereoscopic imaging).

If sufficient data are available, a sensitivity analysis will be performed excluding studies judged to be at high risk of bias.

\section{Grading of evidence}

Certainty of the evidence will be assessed using the Grading of Recommendations, Assessment, Development and Evaluations (GRADE) approach. ${ }^{24}{ }^{25}$ Specifically, prevalence studies will be considered to constitute highcertainty evidence to answer this review question and downgraded for risk of bias, imprecision, inconsistency, indirectness and publication bias. Two reviewers will independently make this judgement. Disagreements will be resolved by discussion, and if required, a third (senior) reviewer will arbitrate.

\section{Patient and public involvement}

There were no time or funds allocated to patient and public involvement, particularly in the context of the current COVID-19 pandemic, so the reviewers were unable to involve patients. However, this systematic review asks an important clinical question and the protocol described follows a standardised approach as per PRISMA-P guidelines. People with prediabetes will be invited to help the reviewers develop a strategy to disseminate the results.

\section{ETHICS AND DISSEMINATION}

This study is a systematic review using aggregated published data, without accessing any personal identifiable information, hence there are no significant ethical or safety concerns. The results of this study will be presented at international conferences and submitted for publication in a peer-reviewed open-access journal. Authors will 
use their networks to encourage broad dissemination of the results.

\section{Author affiliations}

${ }^{1}$ Faculty of Life Sciences and Medicine, King's College London, London, UK

${ }^{2}$ Ophthalmology Research Unit, King's College Hospital NHS Foundation Trust,

London, UK

${ }^{3}$ Division of Diabetes, Endocrinology and Gastroenterology, Institute of Human Development, University of Manchester, Manchester, UK

${ }^{4}$ Pain Research Institute and Department of Cardiovascular \& Metabolic Medicine, Institute of Life Course and Medical Sciences, University of Liverpool and Aintree University Hospital NHS Foundation Trust, Liverpool, UK

${ }^{5}$ Department of Diabetes and Endocrinology, Liverpool University Hospitals NHS Foundation Trust, Liverpool, UK

${ }^{6}$ International Centre for Eye Health, London School of Hygiene and Tropical Medicine, London, UK

${ }^{7}$ Department of Health Data Science, University of Liverpool, Liverpool, UK

${ }^{8}$ Research Division, Weill Cornell Medicine-Qatar, Doha, Qatar

Twitter Jennifer Evans @jevans7 and Sarah Nevitt @sjn_16

Contributors VK, UA and TLJ conceived the review topic. VK performed background exploratory searches and drafted the initial search strategy. VK, JE and PN co-wrote the initial protocol. UA, SN, RAM and TLJ provided critical appraisal and senior oversight of the protocol. For the systematic review, VK and PN will perform the searches, data extraction and analysis. JE will provide oversight of the searches, data analysis and extraction. SN will provide statistical input for data analysis. UA, RAM and TLJ will provide critical appraisal and senior oversight of the final manuscript.

Funding The authors have not declared a specific grant for this research from any funding agency in the public, commercial or not-for-profit sectors.

Competing interests None declared.

Patient consent for publication Not required

Provenance and peer review Not commissioned; externally peer reviewed.

Supplemental material This content has been supplied by the author(s). It has not been vetted by BMJ Publishing Group Limited (BMJ) and may not have been peer-reviewed. Any opinions or recommendations discussed are solely those of the author(s) and are not endorsed by BMJ. BMJ disclaims all liability and responsibility arising from any reliance placed on the content. Where the content includes any translated material, BMJ does not warrant the accuracy and reliability of the translations (including but not limited to local regulations, clinical guidelines, terminology, drug names and drug dosages), and is not responsible for any error and/or omissions arising from translation and adaptation or otherwise.

Open access This is an open access article distributed in accordance with the Creative Commons Attribution Non Commercial (CC BY-NC 4.0) license, which permits others to distribute, remix, adapt, build upon this work non-commercially, and license their derivative works on different terms, provided the original work is properly cited, appropriate credit is given, any changes made indicated, and the use is non-commercial. See: http://creativecommons.org/licenses/by-nc/4.0/.

\section{ORCID iDs}

Varo Kirthi http://orcid.org/0000-0003-4322-1017

Jennifer Evans http://orcid.org/0000-0002-6137-2030

Sarah Nevitt http://orcid.org/0000-0001-9988-2709

Rayaz A Malik http://orcid.org/0000-0002-7188-8903

Timothy L Jackson http://orcid.org/0000-0001-7618-1555

\section{REFERENCES}

1 American Diabetes Association. Diagnosis and classification of diabetes mellitus. Diabetes Care 2010;33 Suppl 1:S62-9.

2 Alberti KG, Zimmet PZ. Definition, diagnosis and classification of diabetes mellitus and its complications. Part 1: diagnosis and classification of diabetes mellitus provisional report of a who consultation. Diabet Med 1998;15:539-53.
3 International Diabetes Federation. Interional diabetes Federation atlas. 9th Edition, 2019. https://www.diabetesatlas.org/

4 Tabák AG, Jokela M, Akbaraly TN, et al. Trajectories of glycaemia, insulin sensitivity, and insulin secretion before diagnosis of type 2 diabetes: an analysis from the Whitehall II study. Lancet 2009;373:2215-21.

5 Vistisen D, Witte DR, Brunner EJ, et al. Risk of cardiovascular disease and death in individuals with prediabetes defined by different criteria: the Whitehall II study. Diabetes Care 2018;41:899-906.

6 Tabák AG, Herder C, Rathmann W, et al. Prediabetes: a high-risk state for diabetes development. Lancet 2012;379:2279-90.

7 Lee CC, Perkins BA, Kayaniyil S, et al. Peripheral neuropathy and nerve dysfunction in individuals at high risk for type 2 diabetes: the promise cohort. Diabetes Care 2015;38:793-800.

8 Azmi S, Ferdousi M, Petropoulos IN, et al. Corneal confocal microscopy identifies small-fiber neuropathy in subjects with impaired glucose tolerance who develop type 2 diabetes. Diabetes Care 2015;38:1502-8.

9 Brownrigg JRW, Hughes CO, Burleigh D, et al. Microvascular disease and risk of cardiovascular events among individuals with type 2 diabetes: a population-level cohort study. Lancet Diabetes Endocrinol 2016;4:588-97.

10 Gong Q, Zhang P, Wang J, et al. Morbidity and mortality after lifestyle intervention for people with impaired glucose tolerance: 30-year results of the dA Qing diabetes prevention outcome study. Lancet Diabetes Endocrinol 2019;7:452-61.

11 Yau JWY, Rogers SL, Kawasaki R, et al. Global prevalence and major risk factors of diabetic retinopathy. Diabetes Care 2012;35:556-64.

12 Cheung N, Mitchell P, Wong TY. Diabetic retinopathy. The Lancet 2010;376:124-36.

13 National Eye Institute. People with diabetes can prevent vision loss, 2019. Available: https://www.nei.nih.gov/sites/default/files/2019-06/ diabetes-prevent-vision-loss.pdf

14 Wong TY, Klein R, Sharrett AR, et al. The prevalence and risk factors of retinal microvascular abnormalities in older persons: the cardiovascular health study. Ophthalmology 2003;110:658-66.

15 Nguyen TT, Wang JJ, Wong TY. Retinal vascular changes in prediabetes and prehypertension: new findings and their research and clinical implications. Diabetes Care 2007;30:2708-15.

16 McGowan J, Sampson M, Salzwedel DM, et al. PRESS Peer Review of Electronic Search Strategies: 2015 Guideline Statement. J Clin Epidemiol 2016;75:40-6.

17 Wilkinson CP, Ferris FL, Klein RE, et al. Proposed International clinical diabetic retinopathy and diabetic macular edema disease severity scales. Ophthalmology 2003;110:1677-82.

18 Alberti KGMM, Eckel RH, Grundy SM, et al. Harmonizing the metabolic syndrome: a joint interim statement of the International Diabetes Federation Task Force on Epidemiology and Prevention; National Heart, Lung, and Blood Institute; American Heart Association; World Heart Federation; International Atherosclerosis Society and International Association for the Study of Obesity. Circulation 2009;120:1640-5.

19 American Diabetes Association. 2. Classification and Diagnosis of Diabetes: Standards of Medical Care in Diabetes-2018. Diabetes Care 2018;41:S13-27.

20 National Cholesterol Education Program (NCEP) Expert Panel on Detection, Evaluation, and Treatment of High Blood Cholestero in Adults (Adult Treatment Panel III). Third report of the National cholesterol education program (NCEP) expert panel on detection, evaluation, and treatment of high blood cholesterol in adults (adult treatment panel III) final report. Circulation 2002;106:3143-421.

21 Yumuk V, Tsigos C, Fried M, et al. European guidelines for obesity management in adults. Obes Facts 2015;8:402-24.

22 Moher D, Liberati A, Tetzlaff J, et al. Preferred reporting items for systematic reviews and meta-analyses: the PRISMA statement. PLoS Med 2009;6:e1000097.

23 Hoy $D$, Brooks $P$, Woolf $A$, et al. Assessing risk of bias in prevalence studies: modification of an existing tool and evidence of interrater agreement. J Clin Epidemiol 2012;65:934-9.

24 Guyatt GH, Oxman AD, Vist GE, et al. GRADE: an emerging consensus on rating quality of evidence and strength of recommendations. BMJ 2008;336:924-6.

25 Iorio A, Spencer FA, Falavigna M, et al. Use of GRADE for assessment of evidence about prognosis: rating confidence in estimates of event rates in broad categories of patients. BMJ 2015;350:h870. 